\title{
Heavy-tailed targets and (ab)normal asymptotics in diffusive motion
}

\author{
Piotr Garbaczewski, Vladimir Stephanovich and Dariusz Kȩdzierski \\ Institute of Physics, University of Opole, 45-052 Opole, Poland
}

\begin{abstract}
We investigate temporal behavior of probability density functions (pdfs) of paradigmatic jumptype and continuous processes that, under confining regimes, share common heavy-tailed asymptotic (target) pdfs. Namely, we have shown that under suitable confinement conditions, the ordinary Fokker-Planck equation may generate non-Gaussian heavy-tailed pdfs (like e.g. Cauchy or more general Lévy stable distribution) in its long time asymptotics. For diffusion-type processes, our main focus is on their transient regimes and specifically the crossover features, when initially infinite number of the pdf moments drops down to a few or none at all. The time-dependence of the variance (if in existence), $\sim t^{\gamma}$ with $0<\gamma<2$, in principle may be interpreted as a signature of sub-, normal or super-diffusive behavior under confining conditions; the exponent $\gamma$ is generically well defined in substantial periods of time. However, there is no indication of any universal time rate hierarchy, due to a proper choice of the driver and/or external potential.
\end{abstract}

PACS numbers: 05.40.Jc, 02.50.Ey, 05.20.-y, 05.10.Gg

\section{INTRODUCTION}

Among a large variety of random walk and primordial noise models/choices, a distinguished role is played by heavy-tailed symmetric Lévy-stable distributions, commonly thought to be an exclusive support for so-called Lévy flights. That view is founded on the concept of independent and identically-distributed random variables of the jump-type and a broad class of infinitely divisible distributions, that are admitted by generalizing the familiar central limit theorem, as encoded in the LévyKhintchine formula. Stable densities generically have no second moments.

The response of Lévy noise to external potentials is most often quantified by means of the Langevin equation with an additive Lévy (stable) driver. The affiliated distribution functions (pdfs) obey so-called fractional Fokker-Planck equations where, in order to handle heavy-tailed pdfs, the (Brownian case) second spatial derivative is replaced by the fractional one of order $0<\mu<2$, c.f. [1]-[10] and references therein. Under confining conditions, the second moments of resultant pdfs may exist and then the temporal behavior of the variance may be employed to quantify (on suitable time scales) sub-, or super-diffusive features of the underlying jump-type dynamics.

In our previous papers $8-10]$ we have noticed that in addition to standard Langevin equation based methods, an alternative modeling approach is worth investigation. It is based on the concept of the Lévy-Schrödinger semigroup-driven dynamics, [8, 12]. Contrary to the familiar (in the context of the Brownian motion) mapping of the Fokker-Planck equation into the Hamiltonian dynamical system, the non-Gaussian case makes a distinction between these two dynamical patterns of behavior. They are inequivalent.

Our departure point was, and still remains, the "stochastic targeting" (also named "reverse engineering") strategy, [1]: given an invariant pdf $\rho_{*}(x)$, design a stochastic jump-type process for which that pres- elected density is a unique asymptotic target. In case of Langevin-driven processes, the basic reconstruction goal amounts to deducing the drift function of the process.

In Refs. [8, 9], given the very same $\rho_{*}(x)$, we have addressed the existence issue of a semigroup-driven dynamics (e.g. the fractional version of the generalized diffusion equation), which relies on the existence of a semigroup potential $\mathcal{V}(x)=-\lambda\left(|\Delta|^{\mu / 2} \rho_{*}^{1 / 2}\right)(x) / \rho_{*}^{1 / 2}(x)$. Since $\rho_{*}(x)$ is presumed to be shared with the Langevindriven Lévy process, we know that it has a non-Gibbsian functional form, [1]. Therefore standard thermalization and (ultimate) thermal equilibrium concepts are invalid in the Lévy context.

In Ref. [10] we have relaxed the common pdf constraint and addressed a fully fledged reconstruction problem for the semigroup dynamics: given an invariant pdf, identify the semigroup-driven Lévy process for which the prescribed pdf $\rho_{*}$ may stand for a unique asymptotic one. Since, to this end, there is no need to invoke the Langevin connection, the ensuing non-Gibbsian obstacle does not appear anymore.

Indeed, under new premises, asymptotic pdfs in the Gibbs form are admissible and a class of jump-type processes, non-trivially responding to environmental inhomogeneities, becomes largely extended to pdfs that are definitely related to Gibbsian thermal equilibria, 10].

The last observation suggests a possibility of a major recasting of the original "reverse engineering" problem of Ref. 1] which was designed to handle jump-type processes only. Namely, while before we have set common invariant pdfs for various jump-type processes, presently we shall consider invariant pdfs that are shared by, seemingly disparate, jump-type (discontinuous) and diffusiontype (continuous) processes. Invariant pdfs that show up a power-law behavior (being e.g. of the inverse polynomial form) are here allowed and thus an issue of a proper thermalization framework (Gibbsian equilibria), that encompasses heavy-tailed distributions, reappears again.

An issue disregarded in the past [13] is that generic Lévy pdfs (like e.g. the familiar Cauchy distribution), 
plainly against casual views, may be embedded in suitable "exponential families" of pdfs [14 16]. A careful exploitation of standard (Shannon) entropy extremum principles, 14, 16], allows to single out a concrete Lévy pdf as a specific exponential family member, at a uniquely defined inverse temperature value.

Anticipating further discussion, let us point out that our major observations are quite general and refer to a broad class of pdfs that are associated with symmetric stable noises and their perturbed (confining regime) versions. It is only an analytical and numerical tractability reason, that makes us mostly to refer to the Cauchy driver in the present paper.

We note that in the heavy-tailed asymptotic regime of diffusion-type processes, the process remains continuous and there is not (albeit appealing) "switch" to any jumping scenario. For all times we deal with a diffusion proceeding in conservative force fields, whose pdf asymptotic features ultimately appear to mimic those normally attributed to jump-type processes.

In a slightly careless manner one may think of a transition "from a diffusive motion to the Lévy flight behavior" . The corresponding transient phenomena (that have been literally interpreted as a transition from a diffusive to jump-type motion) were experimentally recorded in the past [17]. The diffusion vs jump scenario interpretation issue has appeared as well in the semiclassical description of so-called optical lattices, see e.g. [18]-[24].

Apart from an obvious possibility to regard the Lévy driver (and thus to invoke the corresponding nonGaussian probability distribution) as the major random displacement mechanism, it is perhaps less obvious that the Wiener driver actually may give rise to heavy-tailed pdfs as well. Then we encounter the dynamically generated intermediate and/or transitional regimes, where the number of the pdf moments asymptotically drops down to a finite number or none at all.

To elucidate these points, here we employ as a toy model (albeit directly related to the previously mentioned optical lattice issue) a one-parameter family of Cauchy power pdfs, 14]:

$$
\rho_{\alpha}(x)=\frac{\Gamma(\alpha)}{\sqrt{\pi} \Gamma(\alpha-1 / 2)} \frac{1}{\left(1+x^{2}\right)^{\alpha}}, \alpha>1 / 2
$$

as a reference exponential family of pdfs (see e.g. [14]) that comprises the classic Cauchy distribution as its member. This familiy is an exponential one due to a trivial transformatin into the Gibbs-loking function with a logarthmic exponent. (Actually, for each "canonical" Lévy pdf an analogous exponential family embedding can be accomplished.)

The above Cauchy family arises naturally via the standard maximum entropy principle. Namely, one seeks an extremum of the (dimensionless) Shannon entropy $S(\rho)=-\int \rho \ln \rho d x$ of a continuous probability distribution $\rho(x)$ under the constraint that the expectation value $<\ln \left(1+x^{2}\right)>$ takes an a priori prescribed value, [14].

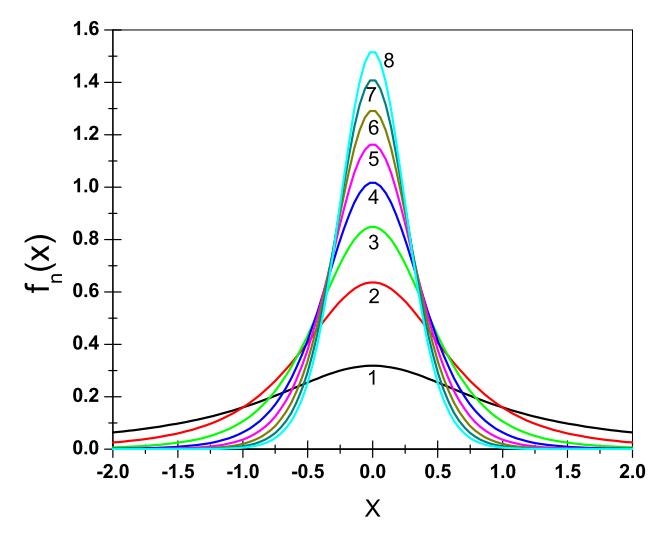

FIG. 1: The members of Cauchy pdf family for $1 \leq n \leq 8$.

If we regard a logarithmic function $\alpha \ln \left(1+x^{2}\right)$ as an external force potential, with $-2 \alpha x /\left(1+x^{2}\right)$ being interpreted as the forward drift in the Langevin equation, then we end up with a familiar Fokker-Planck equation (Wiener driver in action).

A functional form of the force term is identical to that of the physically motivated "cooling force" in optical lattice discussions [21]. With that restoring force, we are capable of generating all members of the above Cauchy family as target pdfs in the large time asymptotic of diffusion-type processes. This linear Fokker-Planck equation option has appeared in the literature before, [21, 22], also in the attempt to interpret the Cauchy family as a family of Tsallis distribution functions [25].

All members of the Cauchy family can be achieved as asymptotic targets for jump-type processes. Both the Lévy-Langevin and Lévy semigroup driven dynamics may be employed to this end. In turn, the same "targeting" is valid if we resort to diffusion-type processes.

An important intrinsic property of the Cauchy family has to do with a number of moments of those pdfs. For a while, let us consider a parameter $\alpha$ in Eq. (1) to assume integer values only: $\alpha \rightarrow n \geq 1$. Then one immediately observes that the Cauchy hierarchy of pdfs, c.f. Fig. 1, involves a monotonically growing number (as parameter $n$ grows from $n=1$ to infinity) of moments of the probability distribution, beginning from none at all for $n=1$. The growing number of moments in existence amounts to an improvement of the pdf localization and entails increasing strength of confinement of Cauchy jumps (e.g. flights), c.f. also [8, 9], if the Cauchy driver is assumed to be in action.

An appealing feature of the Cauchy family is that, even if looking exceptional in many respects, it has received an ample literature coverage not only in the semiclassical analysis of optical lattices [19]-24]. In below, we shall elaborate a bit on another physically important context of Tsallis entropies and related (via entropy maximum principles) Tsallis pdfs. 
Indeed, Cauchy family pdfs were discussed in search for a statistical and thermal (equilibrium) foundation for Tsallis entropies and associated with them probability distributions. That includes asymptotic properties of various Fokker-Planck equations, nonlinear being included, as a dynamical justification for them, see e.g. Refs. [26]-[36]. From a thermal equilibrium point of view, in reference to heavy-tailed pdfs, our approach appears to provide an alternative proposal to that originally formulated in Refs. [26, 28, 29] and [25, 32].

Subsequently we shall discuss in some detail various random motion scenarios that may generate the above Cauchy family of pdfs in their large time asymptotics. After identifying the involved Cauchy semigroup dynamics, we shall demonstrate that diffusion-type processes as well generate heavy-tailed (Cauchy, to be specific) pdfs, in affinity with the case of jump-type processes described by fractional Fokker-Planck equations.

Somewhat surprisingly, it turns out that the conventional diffusive dynamics may be regarded as an alternative (to the fractional one) dynamical model of random behavior that produces non-Gaussian pdfs. This includes heavy tailed ones, that are casually thought to arise exclusively in relation to "free" (we stress the role of independent, identically distributed random displacements) jump-type random motions.

In fact, we demonstrate the validity of the diffusive relaxation scenario from an initial $\delta$ or a Gaussian function to virtually any member of Cauchy power pdf hierarchy (11). In the diffusive dynamics, the resultant pdfs appear to have the Gibbs - Boltzmann form (as should be for the exponential family of pdfs). All those observations readily extend to a broad variety of non-Gaussian pdfs that have been invented in connection with the concept of confined Lévy flights.

Our discussion uncovers an interplay between Lévy and diffusion-type processes that has been left basically ignored in the current "stochastic modeling" literature. We point out that, in principle, one may even not be able to discriminate between these two classes (continuous vs discontinuous) of random processes, if in the vicinity of respective (common for both) equilibrium states. Such observation may be particularly useful to avoid erroneous interpretation, in cases when an experimentally founded data analysis indicates a transient dynamics with a (possibly unusual) crossover behavior, like e.g. that reported in Ref. [17].

In the present paper Langevin noises (Lévy and Wiener driver alike) are considered as additive. For a completeness of exposition, we point out that there are reports in the literature that Langevin equations with the multiplicative (Wiener) noise do give rise to heavy-tailed distributions, see e.g. Ref. [37].

\section{ENTROPY EXTREMUM PRINCIPLES}

\section{A. Shannon vs Tsallis entropies}

An influential Ref. [13] appears to have harmed an open-minded approach to extremum entropy principles, with regard to heavy-tailed probability distribution functions. Its main message has been often uncritically repeated: "the derivation of distributions with inverse power tails from a maximum entropy formalism would be a consequence only of an unconventional auxiliary condition that involves the specification of the average value of a complicated function". This statement has been amplified at the end of Section 2 of Ref. [13]: "It is difficult to imagine that anyone in an a priori manner would introduce a set of auxiliary conditions that could yield the logarithmic term that appears in the (Shannon) entropy function associated with the Lévy distribution." In Ref. [26], the pertinent formula (their Eq. (7)) is called the "ad hoc constraint".

A possible way out of this apparent difficulty has been proposed in the past. It amounts to abandoning the standard Shannon entropy usage in extremum principles and deviate towards non-extensive generalizations of the notion of entropy (effectively one ends up with a nonextensive thermostatistics), so that the heavy-tailed distribution would "more or less naturally" arise. That has been suggested in Refs. [26, 28, 29] and followed in the interpretation of experimental data in Refs. [17]- 24].

We wish to demonstrate that the non-extensive proposal is not the only admissible route, if one looks for mechanisms that generate heavy-tailed target pdfs. The conventional Shannon entropy strategy appears to work satisfactorily as well.

If one seeks for an extremum of the Shannon entropy $S(\rho)=-k_{B} \int \rho \ln (\sigma \rho) d x$ of a continuous probability distribution $\left(k_{B}\right.$ is Boltzmann constant and parameter $\sigma>0$ determines the characteristic length of a system), under the constraint that the expectation value $\int x^{2} \rho(x) d x=\sigma^{2}[26$, an automatic outcome is a Gauss density $\rho_{*}(x)=(\beta / \pi)^{1 / 2} \exp \left(-\beta x^{2}\right)$, where an (implicit) Lagrange multiplier $\beta$ takes the value $\beta=1 / 2 \sigma^{2}$. Finally, the presupposed Gibbs thermal equilibrium condition imposes $\beta=1 / k_{B} T,[26,38]$.

Let us indicate that for generic Gibbsian densities $\rho_{*}(x)=(1 / Z) \exp (-\beta V(x)$, where $V(x)$ stands for an external potential and $Z$ is a normalization factor, the previous moment constraint corresponding to the Lagrange multiplier $\beta$ may readily be generalized to the form $\langle V\rangle=$ const whose equivalent form is $\left\langle\ln \rho_{*}\right\rangle=$ const $^{\prime}$.

So called "ad hoc" constraints of Refs. [13] and [26], albeit with no reference to any effective potential, nor exponential (Gibbs-looking) recasting of the involved pdfs, are nothing more than the demand

$$
\left\langle\ln \rho_{*}\right\rangle=\int \rho(x) \ln \rho_{*}(x) d x=\text { const }
$$

where $\rho_{*}$ stands for any a priori pre-selected Lévy stable 
pdf. C.f. Eq. (21) in Ref. [13] and Eq. (7) in Ref. [26]. We emphasize that the pertinent Lévy distributions are "free noise" models and have nothing to do with the notion of confined Lévy flights.

The proposal of Refs. [26, 28, 29] amounts to considering the new entropy function

$$
S_{q}[\rho]=\frac{k_{B}}{q-1}\left(1-\int d(x / \sigma)[\sigma \rho(x)]^{q}\right)
$$

with a real parameter $q$. An optimization [27] of a suitable likelihood function, under a constraint

$$
\left\langle x^{2}\right\rangle_{q}=\int d(x / \sigma) x^{2}[\sigma \rho(x)]^{q}=\sigma^{2}
$$

results in an extremal pdf:

$$
\rho_{q}(x)=\frac{1}{Z_{q}}\left[1-\beta(1-q) x^{2}\right]^{1 / 1-q}
$$

where

$$
Z_{q}=\left[\frac{\beta(q-1)}{\pi}\right]^{1 / 2} \frac{\Gamma(1 /(q-1))}{\Gamma((3-q) / 2(q-1))}
$$

and we need $1<q<3$ to secure convergence of the normalization integral.

We note that, in view of a direct $q \rightarrow 1$ connection with the Shannon entropy (and the Gibbs density), an interpretation $\beta=1 / k_{B} T$ is enforced. That, in turn, has been a starting point in Refs. [25, 32] to deduce the Tsallis pdf in an asymptotic regime of a well defined Fokker-Planck dynamics. The resultant invariant pdf has the Gibbs form $\rho_{q}(x)=\exp [-\beta V(x)] / Z_{q}$, provided the "external force" potential reads:

$$
V(x)=\frac{1}{\beta(q-1)} \ln \left[1+\beta(q-1) x^{2}\right] .
$$

Let us redefine the involved constants. Namely, after [21] (we modify Eqs. (4) therein), let us set

$$
\beta=\frac{\alpha}{2 D}, \quad q=1+\frac{2 D}{\sigma^{2} \alpha} .
$$

That results in the $V(x)=\sigma^{2}\left[1+(x / \sigma)^{2}\right]$ and $\rho_{q}(x)$ effectively turn over into:

$$
\rho_{q}(x)=\frac{1}{Z_{q}}\left[1+(x / \sigma)^{2}\right]^{-\beta \sigma^{2}},
$$

which differs from Eq. (11) by a trivial replacement of the exponent $\alpha$ by $\beta \sigma^{2}$ and an explicit usage of dimensionless argument $x / \sigma$. Clearly, if $\beta$ plays the role of $1 / k_{B} T$, the signature of thermal equilibrium needs to be directly transferrable to the exponent $\alpha$ as well.

\section{B. Shannon entropy extremum for random systems in logarithmic potentials}

Cauchy family pdfs are labeled by a continuous parameter $\alpha>1 / 2$, whose physical meaning seems to be obscure. In addition to considerations of the previous subsection we shall give more arguments to the contrary. Even without explicitly relying on the optical lattice contexts, [19]-24].

At this point we invoke a classification of maximum entropy principles (MEP) as given in Ref. [14]. Let us look for pdfs that derive from so-called first inverse MEP: given a pdf $\rho(x)$, choose an appropriate set of constraints such that $\rho(x)$ is obtained if Shannon measure of entropy is maximized (strictly speaking, extremized) subject to those constraints.

Namely if a system evolves in a potential $V(x)$ (at the moment, we consider a coordinate $x$ to be dimensionless), we can introduce the following functional

$$
L\{\rho(x)\}=-\lambda \int_{-\infty}^{\infty} V(x) \rho(x) d x-\int_{-\infty}^{\infty} \rho(x) \ln [\rho(x)] d x .
$$

The first term comprises the mean value of a potential. A constant $\lambda$ is (as yet physically unidentified) Lagrange multiplier, which takes care of aforementioned constraints. The second term stands for Shannon entropy of a continuous (dimensionless) pdf $\rho(x)$. An extremum of the functional $L\{\rho(x)\}$ can be found by means of standard variational arguments and gives rise to the following general form of an extremizing pdf $\rho_{*}(x)$ : $\rho_{*}(x)=C \exp (-\lambda V(x))$ which, if regarded as the GibbsBoltzmann pdf, implies that the parameter $\lambda$ can be interpreted as inverse temperature, $\lambda=\left(k_{B} T\right)^{-1}$, at which a state of equilibrium (asymptotic pdf) is reached by a random dynamical system in a confining potential $V(x)$.

A deceivingly simple question has been posed in chap. 8.2.4 of Ref. 14]. Having dimensionless logarithmic potential $\mathcal{V}(x)=\ln \left(1+x^{2}\right)$, one should begin with evaluating a mean value $\mathcal{U}=\langle\mathcal{V}\rangle \equiv \int_{-\infty}^{\infty} \mathcal{V}(x) \rho(x) d x$. Next one needs to show that only if this particular value is prescribed in the above MEP procedure, Cauchy distribution will ultimately arise. Additionally, one should answer what kind of distribution would arise if any other positive expectation value is chosen. The answer proves not to be that straightforward and we shall analyze this issue below.

To handle the problem we admit all pdfs $\rho(x)$ for which the mean value $\left\langle\ln \left(1+x^{2}\right)\right\rangle$ exists, i.e. takes whatever finite positive value. Then, we adopt the previous variational procedure with the use of Lagrange multipliers. This procedure shows that what we extremize is not the (Shannon) entropy itself, but a functional $\mathcal{F}$ with a clear thermodynamic connotation (Helmholtz free energy ana$\log )$ :

$$
\Phi(x)=\alpha \mathcal{V}(x)+\ln \rho(x) \rightarrow \mathcal{F}=\langle\Phi\rangle=\alpha\langle\mathcal{V}\rangle-\mathcal{S}(\rho) .
$$


Here $\mathcal{S}(\rho)=-\langle\ln \rho\rangle$ and $\alpha$ is a Lagrange multiplier. From now on, we consider an exponent $\alpha$ in the form

$$
\alpha=\epsilon_{0} /\left(k_{B} T\right)
$$

where $\epsilon_{0}$ is a characteristic energy scale of a system. Note that here we encounter a dimensionless version of a familiar formula $F=U-T S$, relating the Helmholtz free energy $F$, internal energy $U$ and entropy $S$ of a random dynamical system.

The extremum condition $\delta \mathcal{F}(\rho) / \delta \rho=0$ yields an extremizing pdf in the form $\rho_{\alpha}(x)=\left(1 / Z_{\alpha}\right)\left(1+x^{2}\right)^{-\alpha}$, provided the normalization factor $Z_{\alpha}=\int_{-\infty}^{\infty}\left(1+x^{2}\right)^{-\alpha} d x$ exists. It turns out that the integral can be evaluated explicitly in terms of $\Gamma$ - functions for all $\alpha>1 / 2$, ending up at the previously introduced one-parameter Cauchy family of pdfs (1).

To complete an extremum procedure we can in principle deduce a numerical value of the (Lagrange) parameter $\alpha$, by resorting to our assumption that the mean value $\langle\mathcal{V}\rangle_{\alpha}$ has actually been a priori fixed at a concrete value. This route is not at all straightforward.

To identify the values of above $\alpha$, we need an explicit expression for the mean value

$$
\mathcal{U}_{\alpha}=\langle\mathcal{V}\rangle_{\alpha}=\frac{\Gamma(\alpha)}{\sqrt{\pi} \Gamma(\alpha-1 / 2)} \int_{-\infty}^{\infty} \frac{\ln \left(1+x^{2}\right)}{\left(1+x^{2}\right)^{\alpha}} d x
$$

It turns out that it can be given in terms of the digamma function $\psi(x)=d(\ln \Gamma) / d x$ :

$$
\mathcal{U}_{\alpha}=-\frac{2 \pi}{\sin (2 \pi \alpha)}+\psi(1-\alpha)-\psi\left(\frac{3}{2}-\alpha\right), \alpha>\frac{1}{2} .
$$

This function is divergent at $\alpha=1 / 2$ (see also below) and decays monotonously at large $\alpha$. This decay is conrolled by an asymptotic expansion

$$
\mathcal{U}_{\alpha} \approx \frac{1}{2 \alpha}+\frac{3}{8 \alpha^{2}}+\frac{1}{4 \alpha^{3}}+\ldots
$$

The decay of $\mathcal{U}_{\alpha}$ at large $\alpha$ obeys the inverse power law. It follows that the expansion (15) actually gives a very good approximation of $\mathcal{U}_{\alpha}$ for $\alpha>3$.

We note that, apparently, Eq. (14) involves another divergence problem, if we choose integer $\alpha$. This obstacle can be circumvented by transforming Eq. (14) to an equivalent form that has no (effectively removable) divergencies. Namely, we get

$$
\mathcal{U}_{\alpha}=-\pi \tan \pi \alpha+\psi(\alpha)-\psi\left(\frac{3}{2}-\alpha\right)
$$

and the tangent contribution vanishes for integer $\alpha$. On the other hand, this expression shows that the divergence of $\mathcal{U}_{\alpha}$ at $\alpha \rightarrow 1 / 2$ originates from the first term in (16), as $\psi$ functions have finite values at this point. Near $\alpha=1 / 2$ the first term of Eq. (16) diverges as $(\alpha-1 / 2)^{-1}$.

With an explicit expression for Cauchy family pdfs in hands, we readily evaluate Shannon entropy to obtain

$$
\mathcal{S}_{\alpha}=-\int_{-\infty}^{\infty} \rho_{\alpha}(x) \ln \rho_{\alpha}(x) d x=\ln Z_{\alpha}+\alpha \mathcal{U}_{\alpha}
$$

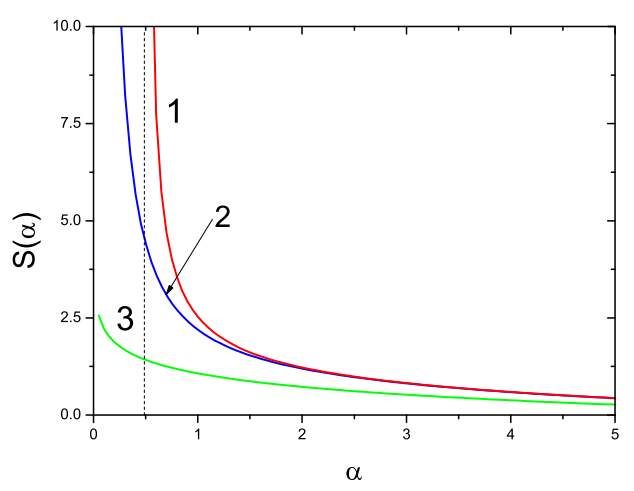

FIG. 2: $\mathcal{S}_{\alpha}$ for Cauchy family (curve 1) and its asymptotic expansion at large $\alpha$ (curve 2). $\mathcal{S}_{\alpha}^{G}$ for Gaussian family is also shown (curve 3 ).

Then, the (as yet dimensionless) Helmholtz free energy $\mathcal{F}_{\alpha}$ reads

$$
\mathcal{F}_{\alpha}=\alpha \mathcal{U}_{\alpha}-\mathcal{S}_{\alpha} \equiv-\ln Z_{\alpha},
$$

with $\alpha$ being the dimensionless analog of the inverse temperature.

We note, that in view of the divergence of $Z_{\alpha}$, both the Shannon entropy and the Helmholtz free energy (likewise $\mathcal{U}_{\alpha}$ ) cease to exist at $\alpha=1 / 2$. We plot $\mathcal{S}_{\alpha}$ as a function of $\alpha$ in Fig 2 It is seen that entropy monotonously decays for $\alpha>1 / 2$ and for larger values of $\alpha$. An asymptotic expansion of the entropy shows logarithmic plus inverse power signatures

$$
\mathcal{S}_{\alpha} \approx \frac{1}{2}\left(1-\ln \frac{\alpha}{\pi}\right)+\frac{3}{4 \alpha}+\frac{3}{8 \alpha^{2}}+\ldots
$$

These series are shown along with the entropy in Fig. 2.

As $\alpha$ grows, the number of moments of respective pdfs increases. That allows to expect that an "almost Gaussian" behavior should be displayed by $\alpha \gg 1$ members of Cauchy family. This is indeed the case as discussed in Section II.G.

\section{Thermalization via Fokker-Planck dynamics}

Let us now show that the variational principle explicitly identifies an equilibrium solution of the FokkerPlanck equation for standard Smoluchowski diffusion processes. To address our thermalization issue correctly, we now use dimensional units. The Fokker - Planck equation that drives an initial probability density $\rho(x, t=0)$ to its final (equilibrium) form $\rho(x, t \rightarrow \infty)$ reads

$$
\partial_{t} \rho=D \Delta \rho-\nabla \cdot(b(x) \rho) .
$$

Here, the drift field $b(x)$ is time-independent and conservative, $b(x)=-\nabla V(x) /(m \gamma)(V(x)$ is a potential, while 

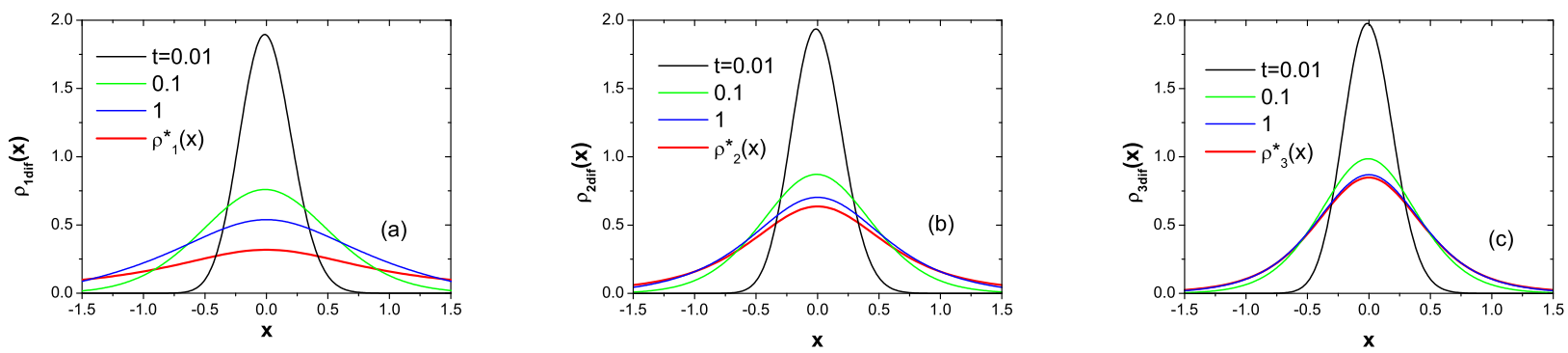

FIG. 3: Time evolution of pdf's $\rho(x, t)$ for Smoluchowski processes in logarithmic potential $\ln \left(1+x^{2}\right)$. The initial $(t=0)$ pdf is set to be a Gaussian with height 25 and half-width $\sim 10^{-3}$. The first depicted stage of evolution corresponds to $t=0.01$. Target pdfs are the members of Cauchy family for $\alpha=1$ (panel (a)), 2 (panel (b)) and 3 (panel (c)) respectively.

$m$ is a mass and $\gamma$ is a reciprocal relaxation time of a system). We keep in mind that $\rho$ and $b \rho$ vanish at spatial infinities or other integration interval borders.

If Einstein fluctuation-dissipation relation $D=$ $k_{B} T / m \gamma$ holds, the equation (20) can be identically rewritten in the form $\partial_{t} \rho=\nabla[\rho \nabla \Psi] /(m \gamma)$, where

$$
\Psi=V+k_{B} T \ln \rho
$$

whose mean value is indeed the Helmholtz free energy of random motion

$$
F \equiv\langle\Psi\rangle=U-T S .
$$

Here the (Gibbs) entropy reads $S=k_{B} \mathcal{S}$, while an internal energy is $U=\langle V\rangle$. In view of assumed boundary restrictions at spatial infinities, we have $\dot{F}=-(m \gamma)\left\langle v^{2}\right\rangle \leq$ 0 , where $v=-\nabla \Psi /(m \gamma)$. Hence, $F$ decreases as a function of time towards its minimum $F_{*}$, or remains constant.

Let us consider the stationary (large time asymptotic) regime associated with an invariant density $\rho_{*}$ (c.f. Ref. [39] for an extended discussion of that issue). Then, $\partial_{t} \rho=0$ and we have $\nabla \Psi\left[\rho_{*}\right]=C \rho_{*}(C$ is arbitrary constant) which yields $\rho_{*}=(1 / Z) \exp \left[-V / k_{B} T\right]$. Therefore, at equilibrium:

$$
\Psi_{*}=V+k_{B} T \ln \rho_{*} \Longrightarrow\left\langle\Psi_{*}\right\rangle=-k_{B} T \ln Z \equiv F_{*},
$$

to be compared with Eq. (18). Here, the partition function equals $Z=\int \exp \left(-V / k_{B} T\right) d x$, provided that the integral is convergent. Since $Z=\exp \left(-F_{*} / k_{B} T\right)$ we have recast $\rho_{*}(x)$ in the familiar Gibbs-Boltzmann form $\rho_{*}=\exp \left[\left(F_{*}-V\right) / k_{B} T\right]$.

On physical grounds, $V(x)$ carries dimensions of energy. Therefore to establish a physically justifiable thermodynamic picture of Smoluchowski diffusion processes, relaxing to Cauchy family pdfs in their large time asymptotics, we need to assume that logarithmic potentials $\mathcal{V}(x)=\ln \left(1+x^{2}\right)$ are dimensionally scaled to the form $V(x)=\epsilon_{0} \mathcal{V}$ where $\epsilon_{0}$ is an arbitrary constant with physical dimensions of energy.

By employing $\left(1 / \epsilon_{0}\right) V(x)=\ln \left(1+x^{2}\right)$, we can recast previous variational arguments (MEP procedure) in terms of dimensional thermodynamical functions. Namely, in view of $k_{B} T \Phi(x)=V(x)+k_{B} T \ln \rho(x)$ we have an obvious transformation of Eqs. (11) and (18) into Eqs. (22) and (23) respectively with $k_{B} T \Phi(x)=\Psi(x)$.

The above dimensional arguments tell us that in confining logarithmic potentials, Cauchy family of pdfs can be regarded as a one-parameter family of equilibrium pdfs, where the reservoir temperature $T$ enters through the exponent $\alpha$. Proceeding in this vein, we note that $\epsilon_{0}$ should be regarded as a characteristic energy (energy scale) of the considered random system.

We observe that $\alpha \rightarrow \infty$ corresponds to $T \rightarrow 0$ i.e. a maximal localization (Dirac delta limit) of the corresponding pdf. The opposite limiting case $\alpha \rightarrow 1 / 2$ looks interesting. Namely, we have $\mathcal{S}(\alpha \rightarrow 1 / 2) \rightarrow \infty$. To grasp the meaning of this limiting regime, we rewrite $\alpha=1 / 2$ in the form $k_{B} T=2 \epsilon_{0}$. Accordingly, the temperature scale, within which our system may at all be set at thermal equilibrium, is bounded: $0<k_{B} T<2 \epsilon_{0}$. For temperatures exceeding the upper bound $T_{\max }=2 \epsilon_{0} / k_{B}$ no thermal equilibrium is possible in the presence of (weakly, i.e. weaker then, e.g., $V(x) \sim x^{2}$ ) confining logarithmic potentials $V(x)=\epsilon_{0} \ln \left(1+x^{2}\right)$. The case of $\alpha=1$ i.e. $k_{B} T=\epsilon_{0}$ corresponds to Cauchy density.

For clarity of presentation (dynamical interpolation scenarios between initial Gaussian and equilibrium Cauchy-type pdfs do not seem to have ever been explicitly considered in the literature), in Fig. 3 we plot various stages of the diffusive Fokker - Planck dynamics for processes that all have been started from a narrow Gaussian. The resultant (large time asymptotic) equilibrium pdfs are members of the Cauchy family, labeled respectively by $\alpha=1,2$ and 3 .

\section{CAUCHY FAMILY TARGETING: TRANSIENT DYNAMICS AND RELAXATION PATTERNS}

To make the paper self-contained, here we describe the dynamics of probability distributions $\rho(x, t)$, whose time evolution is started from the Dirac delta-type initial data 
(actually, a very narrow Gaussian), but might be driven by three different stochastic mechanisms. Apart form the common initial data choice, we shall demonstrate that a common target (invariant) pdf to which the pertinent processes relax in the large time asymptotic, is shared by:

(i) diffusion-type process with a well defined drift function $b_{\text {drift }}(x)$ and the Wiener driver in action,

(ii) Langevin-driven jump type process, with a suitable drift function $b_{\text {jump }}(x)$ and the Cauchy driver in action, (iii) Lévy-Schrödinger semigroup-driven jump type process of Ref. [8], with an (effective) semigroup potential $\mathcal{V}(x)$ and the Cauchy driver implicit.

The above three patterns of dynamical behavior will be demonstrated by assuming that asymptotic (target) pdfs are members of the Cauchy family

$$
\rho_{* n}=\frac{A_{n}}{\left(1+x^{2}\right)^{n}}, A_{n}=\frac{(n-1) !}{\sqrt{\pi} \Gamma(n-1 / 2)} .
$$

It turns out that for our purposes it suffices to investigate the above dynamics for $1 \leq n \leq 4$ only, the dynamics for higher $n$ being a repetition of the established patterns of behavior.

Since we require processes (i)-(iii) to share an asymptotic pdf $\rho_{* n}$ for each choice of $n$, the semigroup potentials (and drift functions, if applicable) will certainly differ from case to case. An additional technical input that needs to be mentioned in view of a common initial pdf (actually, an "almost" Dirac delta) for all considered processes, is that we refer to the well developed theory of so-called Schrödinger boundary data and stochastic interpolation problem, [11, 12]. That allows to devise Markovian dynamics scenarios which interpolate between a priori prescribed initial and terminal (eventually those referring to the large time asymptotic and relaxation regimes) pdf data.

\section{A. Cauchy target, $\rho_{* 1}$.}

Here and subsequently, for notational and computational simplicity, we shall get rid of all freely adjustable parameters (like e.g. $\beta, \gamma$, see e.g. [8]) and set them equal one. The familiar Cauchy pdf:

$$
\rho_{* 1}=\frac{1}{\pi\left(1+x^{2}\right)} .
$$

is known to be a stationary pdf of the OrnsteinUhlenbeck-Cauchy (OUC) stochastic process, [8, 12].

\section{Cauchy driver: jump-type process}

Following a widely accepted reasoning we take for granted that the Langevin equation, with additive deterministic and Lévy noise terms, gives rise to the fractional Fokker-Planck equation, whose form faithfully parallels the Brownian version: $\dot{x}=b(x)+A^{\mu}(t) \Longrightarrow \partial_{t} \rho=$ $-\nabla(b \rho)-\lambda|\Delta|^{\mu / 2} \rho$. All notations are consistent with those of Refs. [8, 9, 12]. To deal with the Cauchy driver, we set $\mu=1$.

Cauchy pdf (25) is a stationary pdf of the Langevindriven fractional Fokker-Planck equation, with noise intensity $\lambda=1$

$$
\partial_{t} \rho=-\nabla(b \rho)-|\Delta|^{1 / 2} \rho .
$$

Since the process is by construction relaxing to an invariant pdf $\rho_{*}(x)$, the drift function is defined as follows

$$
b(x)=b_{j u m p}(x)=-\frac{1}{\rho_{*}(x)} \int\left(|\nabla| \rho_{*}\right)(x) d x .
$$

Accordingly, by selecting the pdf (25), to which the OUC process actually relaxes, we identify the corresponding drift function as $b_{1, j u m p}(x)=-x$.

\section{Semigroup dynamics with Cauchy driver}

In Ref. [8] we have investigated in some detail the Cauchy-Schrödinger semigroup-driven random motion scenario (e.g. semigroup dynamics with Cauchy driver). In this theoretical framework, the major ingredient of the formalism is the (pseudo-differential, self-adjoint in a suitable Hilbert space) Hamiltonian operator $\hat{H}_{\mu} \doteq$ $\lambda|\Delta|^{\mu / 2}+\mathcal{V}$, that gives rise (presently, we set $\mu=1=\lambda$ ) to a fractional analog of the familiar generalized diffusion equation, c.f. [9],

$$
\partial_{t} \Psi=-|\Delta|^{1 / 2} \Psi-\mathcal{V} \Psi .
$$

The dynamics of the related pdf

$$
\rho(x, t)=\Psi(x, t) \rho_{*}^{1 / 2}(x)
$$

is fully determined by Eq. (28), provided $\rho_{*}$ stands for an a priori given terminal (asymptotic) probability density of the involved stochastic process.

The effective potential $\mathcal{V}(x)$ for the semigroup-driven jump-type process reads

$$
\mathcal{V}(x)=-\frac{|\nabla| \rho_{*}^{1 / 2}}{\rho_{*}^{1 / 2}} .
$$

Presently, we identify $\rho_{*}$ with $\rho_{* 1}$. A functional form of the corresponding $\mathcal{V}(x)$ has been found in Ref. [8]:

$$
\mathcal{V}_{1}(x)=\frac{1}{\pi}\left[-\frac{2}{\sqrt{a(x)}}+\frac{x}{a(x)} \ln \frac{\sqrt{a(x)}+x}{\sqrt{a(x)}-x}\right] \text {. }
$$

where $a(x)=1+x^{2}$, c.f. also Fig. 1 in Ref. [8].

The temporal behavior of the Langevin-driven and semigroup driven process with $\rho_{* 1}(x)$ as the asymptotic pdf, has been visualized earlier in Figs 1 and 2 of Ref. [10]. Clearly, the semigroup potential $\mathcal{V}_{1}(x)$ is completely divorced from the harmonic potential $V(x)=x^{2} / 2 \rightarrow$ $-\nabla V=-x=b_{1, j u m p}(x)$, appropriate for the Langevindriven OUC process. We observe that $\rho_{* 1}(x)$ cannot be rewritten in the Gibbs form $(1 / Z) \exp [-V(x)]$, in conformity with arguments of Ref. [1]. 


\section{Wiener driver: diffusion-type process}

The previous two dynamical patterns of behavior seem to be natural in connection with the Cauchy density, since both refer to the Cauchy noise (e.g. driver) and the Cauchy operator $|\nabla|=|\Delta|^{1 / 2}$ as the generator of random motion. Therefore, it seems to be far from being obvious that we can address the previous stochastic interpolation issue in terms of diffusion-type processes, hence with the Wiener driver in action.

For a diffusion process that is governed by the standard Fokker-Planck equation

$$
\partial_{t} \rho=\frac{1}{2} \Delta \rho-\nabla(b \cdot \rho)
$$

with a certain $\rho_{*}(x)$ as its stationary solution, at least on formal grounds we can identify the drift of the process $b(x)$ as

$$
b=b_{\text {diff }}=\frac{1}{2} \nabla \ln \rho_{*} .
$$

Consequently, upon assuming that $b_{d i f f}(x)=-\nabla V_{*}$, we have $\rho_{*}(x)=(1 / Z) \exp \left[-V_{*}(x) / k_{B} T\right]$, where $1 / Z$ is a normalization constant. This has the familiar GibbsBoltzmann form, although $V_{*}(x)$ surely has nothing in common with $V(x)$ appropriate for the Cauchy-Langevin dynamics.

Indeed, under the diffusion (i.e. Langevin-driven) process premises,

$$
\dot{x}=b(x, t)+A(t)
$$

where $\langle A(s)\rangle=0,\left\langle A(s) A\left(s^{\prime}\right)\right\rangle=\delta\left(s-s^{\prime}\right)$, a demand that an asymptotic pdf is in the Cauchy form Eq. (25), enforces the drift function

$$
b_{1, \operatorname{diff}}(x)=-\frac{2 x}{1+x^{2}},
$$

which is a gradient field with the potential $V_{* 1}(x)=$ $\ln \left(1+x^{2}\right)$. These expressions have been substituted to the corresponding numerical routines to obtain a computerassisted picture of the time evolution $\rho_{1}(x, t)$ and get confirmed that such diffusion processes are consistent.

A comparison of the above three different (CauchyLangevin, Cauchy semigroup and Wiener-Langevin) stochastic interpolation scenarios, all started form identical initial data (Dirac delta approximation by a Gaussian) data and all terminating (in the large time asymptotic) at common for all Cauchy pdf, is provided in Fig. (4

The diffusion-type dynamics is faster than that of the Langevin-driven jump-type process. On the other hand, to achieve the target pdf, the semigroup dynamics needs almost twice a time $(t=15)$ as that $(t=8)$ needed for Cauchy - Langevin relaxation. In Fig. 4, we have indicated time instants at which the simulated pdf, within the resolution quality of the figures, cannot be distinguished from the target $\operatorname{pdf} \rho_{* 1}$.
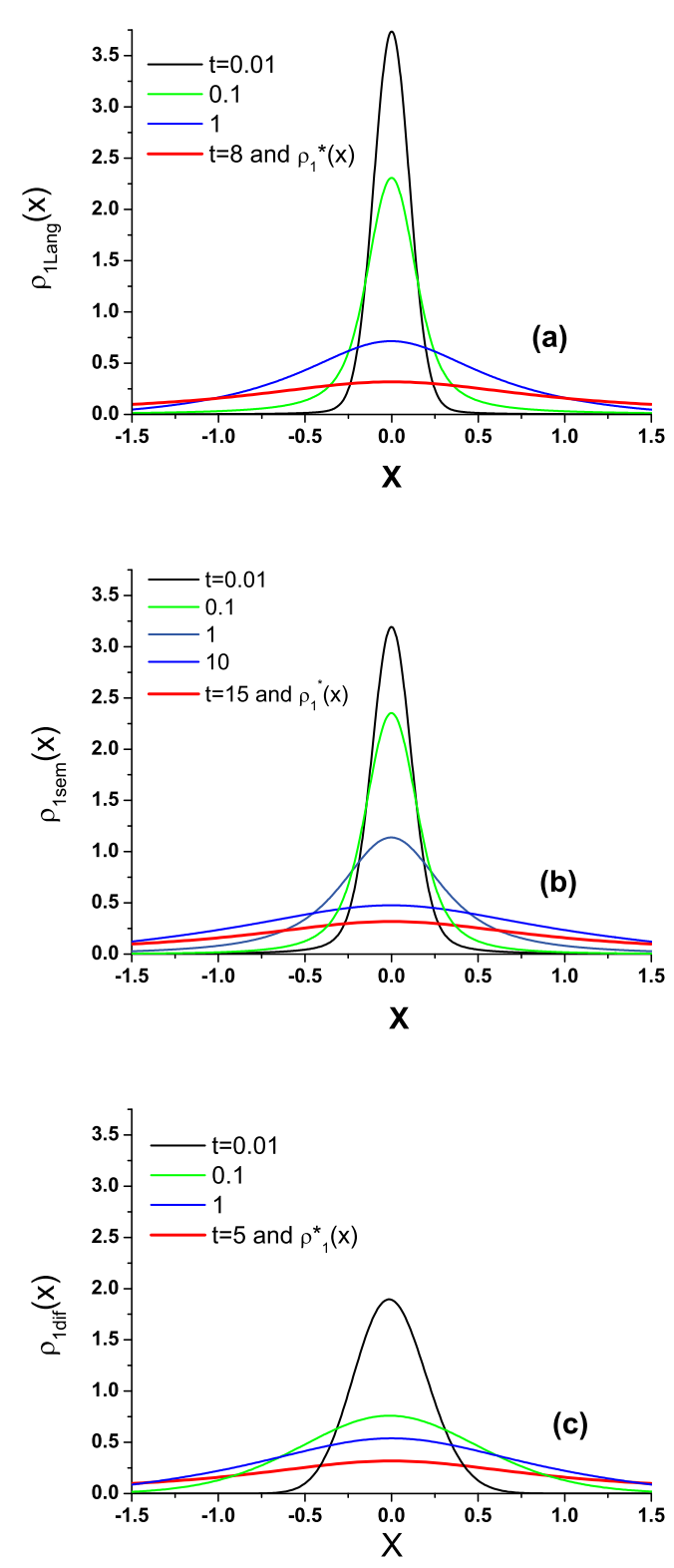

FIG. 4: Time evolution of pdf's $\rho(x, t)$ for the CauchyLangevin dynamics (panel (a)), Cauchy-semigroup-induced evolution (panel (b)) and the Wiener-Langevin process (panel (c)). The common target pdf is the Cauchy density, while the initial $t=0 \mathrm{pdf}$ is set to be a Gaussian with height 25 and half-width $\sim 10^{-3}$. The first depicted stage of evolution corresponds to $t=0.01$. The time rate hierarchy seems to be set: diffusion being fastest, next Lévy-Langevin and semigroupdriven evolutions being slower than previous two. However the outcome is not universal, as our subsequent discussion will show.

The above relaxation pattern is generic for Cauchy family of pdfs. However, the resulting "speed" hierarchy is not generic for stochastic processes at hand. Below we shall demonstrate that time rates of relaxation for 
our three exemplary processes (i.e. jump-type Langevin, jump-type semigroup, diffusion-type) do not follow any definite hierarchy.

\section{B. Second order target, $\rho_{* 2}$.}

With the basic notation specified, we can proceed in quick steps. Let us consider the $n=2$ pdf of the Cauchy hierarchy

$$
\rho_{* 2}=\frac{2}{\pi\left(1+x^{2}\right)^{2}} .
$$

If regarded as an invariant density of the CauchyLangevin stochastic process, this pdf gives rise to the following drift function

$$
b_{2, j u m p}(x)=-\frac{x}{8}\left(x^{2}-3\right) .
$$

The semigroup-driven process, relaxing to $\rho_{* 2}(x)$ in the large time asymptotics, is determined by specifying the semigroup potential, [8] c.f. Fig. 1 there-in:

$$
\mathcal{V}_{2}(x)=\frac{x^{2}-1}{x^{2}+1}
$$

The affiliated diffusion process (Wiener driver), with the very same asymptotic pdf has the drift function

$$
b_{2, \text { diff }}(x) \equiv 2 b_{1, \text { diff }}(x)=-\frac{4 x}{1+x^{2}} .
$$

At this point, we note a recursive formula $b_{n, \operatorname{diff}}(x) \equiv$ $n b_{1, \text { diff }}(x)$ and $V_{* n}(x)=n \ln \left(1+x^{2}\right)$.

Qualitatively, the temporal behavior of the three involved stochastic processes mimics the pattern of $n=1$, according to Fig. 4. except that with the growth of $n$ the speed with which the processes relax increases.

\section{Third order target, $\rho_{* 3}$.}

The third, $\mathrm{n}=3$ member, of the pdfs family reads

$$
\rho_{* 3}=\frac{8}{3 \pi\left(1+x^{2}\right)^{3}} .
$$

It is an invariant probability density of the CauchyLangevin process with the drift function

$$
b_{3, j u m p}(x)=-\frac{x}{16}\left(15+10 x^{2}+3 x^{4}\right) .
$$

An effective potential for the Cauchy-Schrödinger dynamics can be evaluated by means of the Cauchy principal value integrals. Following methods of Ref. [8] we have arrived at its explicit functional form

$$
\mathcal{V}_{3}(x)=\frac{1}{\pi}\left[\frac{2\left(x^{2}-2\right)}{\sqrt{a(x)}}+\frac{3 x}{a(x)} \ln \frac{\sqrt{a(x)}+x}{\sqrt{a(x)}-x}\right] .
$$

where $a(x)=1+x^{2}$. Like the previous two semigroup potentials, that were bounded from below and above, the present one, although unbounded from above, still fulfills minimal requirements set in the general theoretical framework of Ref. [12]. It is bounded from below by $\mathcal{V}_{3}(0)=-4 / \pi$ and for $|x| \rightarrow \infty$ we have $\mathcal{V}_{3}(x) \approx 2|x| / \pi$.

The diffusion process with $\rho_{* 3}(x)$ as its asymptotic target is characterized by the drift function $b_{3, \text { diff }}(x)=$ $-6 x /\left(1+x^{2}\right)$.

\section{Fourth order target, $\rho_{* 4}$.}

Although we know that nothing illuminating might happen in the qualitative picture of the time evolution, if compared to the previous cases, for completeness we provide drift functions and the semigroup potential, that can be derived if $n=4 \mathrm{pdf}$ is to be asymptotic target of, respectively: Cauchy-Langevin, Cauchy semigroup and diffusive motions:

$$
\rho_{* 4}=\frac{16}{5 \pi\left(1+x^{2}\right)^{4}} .
$$

The Cauchy-Langevin drift presently reads

$$
b_{4, j u m p}(x)=-\frac{x}{16}\left(35+35 x^{2}+21 x^{4}+5 x^{6}\right),
$$

the Cauchy-Schrödinger semigroup potential appears in the form

$$
\mathcal{V}_{4}(x)=\frac{x^{4}+6 x^{2}-3}{2\left(x^{2}+1\right)}
$$

while the drift of an affiliated diffusion process is $b_{4, \operatorname{diff}}(x)=-8 x /\left(1+x^{2}\right)$.

\section{E. Tail (large $|x|)$ regularities}

It is easily seen from the above equations that as $|x| \rightarrow$ $\infty$, we encounter some regularities. Namely, pdfs in the Cauchy hierarchy for large $|x|$ behave like

$$
\rho_{* n}(x) \approx \frac{1}{x^{2 n}} .
$$

Cauchy-Langevin drifts have large $|x|$ asymptotics of the form

$$
b_{n, j u m p}(x \rightarrow \infty) \approx x^{2 n-1} .
$$

Cauchy-Schrödinger semigroup potentials behave like

$$
\begin{aligned}
& \mathcal{V}_{1}(|x| \rightarrow \infty) \approx \frac{2 \ln |x|}{|x|} \sim \frac{1}{|x|}, \\
& \mathcal{V}_{2}(|x| \rightarrow \infty) \approx 1 \equiv|x|^{0}, \\
& \mathcal{V}_{3}(|x| \rightarrow \infty) \approx|x|, \\
& \mathcal{V}_{4}(|x| \rightarrow \infty) \approx|x|^{2},
\end{aligned}
$$


so that for arbitrary $n$

$$
\mathcal{V}_{n}(|x| \rightarrow \infty) \approx|x|^{n-2} .
$$

The forward drifts of a diffusion - type processes, for which Cauchy family pdfs (24) are their respective asymptotic targets, read

$$
b_{n, \operatorname{diff}}(x) \approx-\frac{2 n}{x} .
$$

\section{F. A cross-over between exponential and power law behavior}

We have mentioned before that the Cauchy hierarchy is ordered with respect to an overall number of moments in existence, of the probability distribution. Namely, for $n=1$ even the first moment does not exist. In principle (if Cauchy principal value of the corresponding integral is considered) we can admit the existence of the first moment. But still, the second moment does not exist.

For $n=2$, we have first three moments, the third one exists conditionally if the above Cauchy principal value is considered. Ultimately, each $\rho_{* n}$ has exactly $2 n-1$ moments for each $n \geq 1$.

On the other hand, in our numerical procedures, the nonlinear (in a sense that corresponding drift function is not linear) diffusion - type motion is started from a very narrow Gaussian. As indicated in the caption to Fig. 4, its height (maximum) is $\sim 25$ while half-width $\sim 10^{-3}$. This pdf certainly admits all moments.

In the course of the diffusion - type evolution with a target pdf belonging to the Cauchy family, quite apart from incompatible microscopic mechanisms (jumps vs continuous paths) there must arise a definite cross-over from Gaussian to Cauchy hierarchy behavior. The latter hierarchy exhibits the power law asymptotics to be set against Gaussian (stronger then exponential) decay. Therefore higher moments of the initial pdf, while evolving diffusively, must consecutively disappear in the course of time, if any Cauchy density is to be a target pdf.

To visualize this intriguing crossover between Gaussian and Cauchy family behaviors, we have numerically compared the time development of largest finite moment for a given $n$ (which is $(2 n-2)$-th moment of Cauchy family (24) pdfs) with that of a smallest nonexistent moment (which is $2 n$-th moment of the same family). This evolution has been generated by Cauchy - Langevin driven diffusion-type $\rho(x, t)$ beginning (at $t=0$ ) at above very narrow Gaussian and ending (at $t \rightarrow \infty$ ) at $\rho_{* n}$ (24). This has been done for $n=2-4$ and is reported in Fig. [5.

The special case $n=1$ has been considered separately. Namely, following our earlier results, Ref. [10], we consider the half-width at half-maximum of function (25) instead of the above largest finite moment. Then, similar to the above $n>1$ case, we compare the time evolution of this quantity in the course of the OUC process, with that of the second moment for a diffusion-type probability distribution $\rho(x, t)$ having $\rho_{* 1}(x)$ (25) as the target (asymptotic) pdf, see Fig. 5a

\section{G. Interpolation between Cauchy and Gaussian distributions}

Since, within the Cauchy hierarchy, the number of moments increases with $n$, we can justifiably ask for its link with the Gaussian pdf. At least, for large $n$.

Let us rewrite Eq. (1) in the form

$$
\rho_{\alpha}(x)=\frac{A_{\alpha}}{\left(1+x^{2}\right)^{\alpha}} \equiv \exp \left(\ln \rho_{\alpha}\right)
$$

where $\ln \rho_{\alpha}=\ln A_{\alpha}-\alpha \ln \left(1+x^{2}\right)$.

We observe that the limiting procedure $\alpha \rightarrow \infty$ enforces strong localization properties of the pdf so that only a small vicinity of $x=0$ matters. Indeed, at large $\alpha$, the pdf tails become very steep and do not play a decisive role. This motivates the following series expansions

$$
\begin{aligned}
\ln (1+y) & =y-\frac{y^{2}}{2}+\frac{y^{3}}{3}-\frac{y^{4}}{4}+\ldots, y=x^{2}, \\
A_{\alpha}(\alpha \rightarrow \infty) & =\sqrt{\frac{\alpha}{\pi}}-\frac{3}{8 \sqrt{\pi \alpha}}-\frac{7}{128 \alpha \sqrt{\pi \alpha}}-. .
\end{aligned}
$$

Accordingly:

$$
\begin{gathered}
\rho_{\alpha}(x, \alpha \gg 1) \approx\left[\sqrt{\frac{\alpha}{\pi}}-\frac{3}{8 \sqrt{\pi \alpha}}-\frac{7}{128 \alpha \sqrt{\pi \alpha}}-. .\right] \times \\
\exp \left(-\alpha x^{2}+\alpha \frac{x^{4}}{2}-. .\right),
\end{gathered}
$$

It is obvious that leading terms (in the large $\alpha$ regime) coincide with a perfect Gaussian

$$
\rho_{\alpha}(x, \alpha \gg 1) \approx \sqrt{\frac{\alpha}{\pi}} \exp \left(-\alpha x^{2}\right)
$$

We can easily check that function (58) is normalized. Moreover, Eq. (57) sets a "bridge" between the Gaussian and large $\alpha$ members of the Cauchy family. The account of a sufficiently large number of terms in the series (57), permits to control an accuracy of the "Gaussian approximation" of Cauchy family pdfs with large $\alpha$. Ultimately, since $\alpha \rightarrow \infty$ stands for a sequential approximation of the Dirac delta functional, our limiting procedure within the Cauchy family provides another sequential approximation of the Dirac delta. 


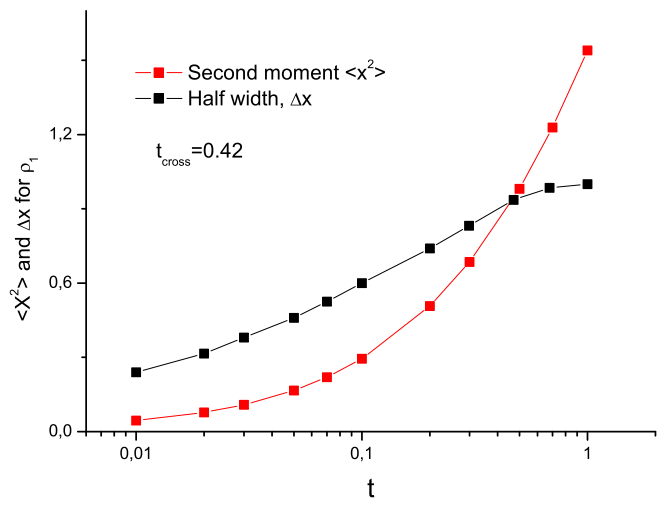

(a) Half-width and $\left\langle X^{2}\right\rangle$ (divergent) for $\rho_{* 1}$

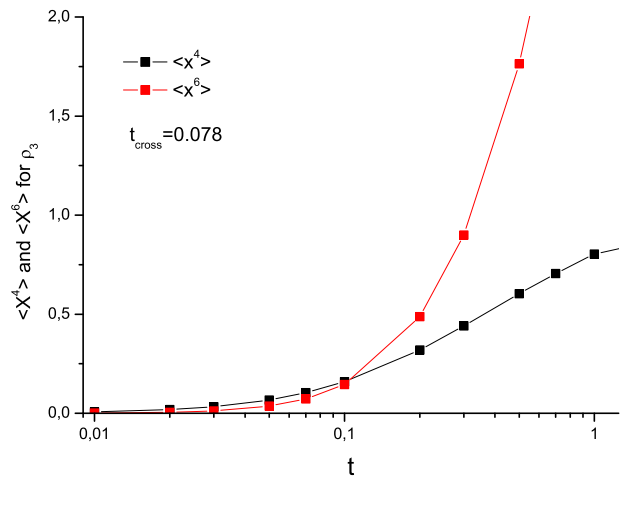

(c) $<X^{4}>$ and $<X^{6}>$ (divergent) for $\rho_{* 3}$

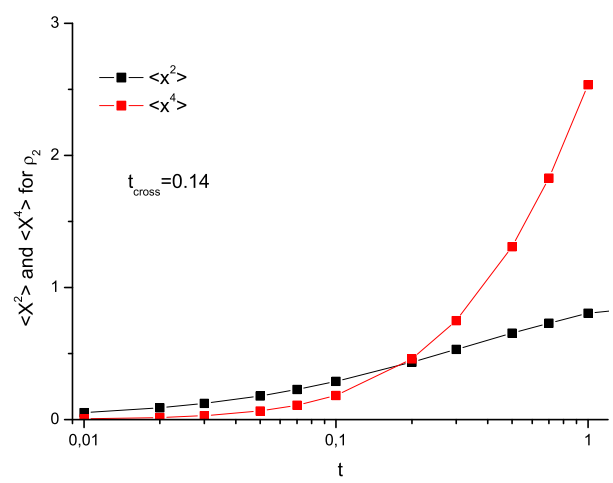

(b) $<X^{2}>$ and $<X^{4}>$ (divergent) for $\rho_{* 2}$

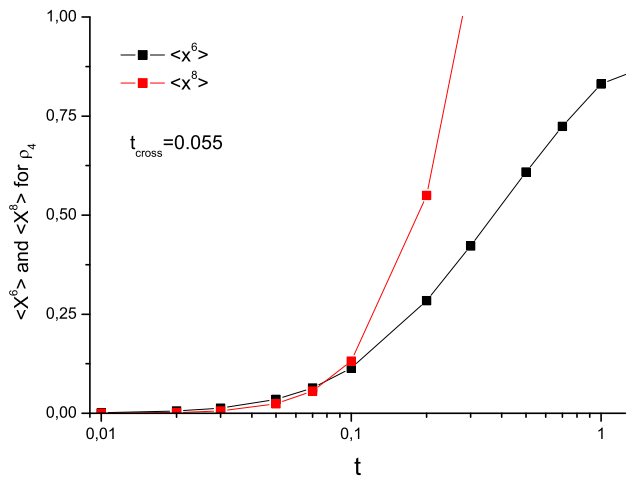

(d) $<X^{6}>$ and $\left\langle X^{8}>\right.$ (divergent) for $\rho_{* 4}$

FIG. 5: The time evolution of the last convergent and first divergent moment for pdfs $\rho_{* 1}-\rho_{* 4}$, shown on panels (a) - (d) respectively. Crossover time instants are shown on the corresponding panels.

\section{GENERIC RELAXATION PATTERNS}

\section{A. Regularity conditions in stochastic modeling}

\section{Jeopardies}

The present subsection is motivated by various regularity conditions that guarantee the existence and uniqueness of solutions, both for stochastic differential equations and Fokker-Planck equations (fractional being included), see Refs. 39 42] and [2, 3]. Those mathematical restrictions do not seem to worry physics-oriented practitioners and we shall indicate why in some cases this disregard might be justified.

A transparent picture of jeopardies to be met, comes within the traditional Brownian motion framework. There, drifts and so-called diffusion functions (in our case, they reduce to diffusion coefficients) need to obey the Lipschitz and growth bounds, [39, 40]. It is easy to generate Langevin and Fokker-Planck equations with drifts that violate the growth condition. Then, some care is necessary, because the general theory tells us that the stochastic process in question may explode in a finite time, [40].

Polynomial drift functions typically violate the growth condition. We shall analyze a possible significance of this violation, by employing: (i) numericallly generated solution of the Fokker-Planck equation, (ii) trajectory wise Monte Carlo (MC) simulations of pdfs, in terms of Langevin equation-generated bunch (c.a. 105) of Brownian paths.

We demonstrate that, despite the violation of the growth condition, no hint of a possible explosion is detected and the dynamical pattern of behavior remains regular up to arbitrarily large times, available in our numerical simulations, both in FPE solution and in direct $\mathrm{MC}$ simulations. Note, that in preceding discussion we have already invoked the FPE (both standard and fractional) with polynomial drifts. A number of examples can also be found in Refs. [5, 7, 39]. 

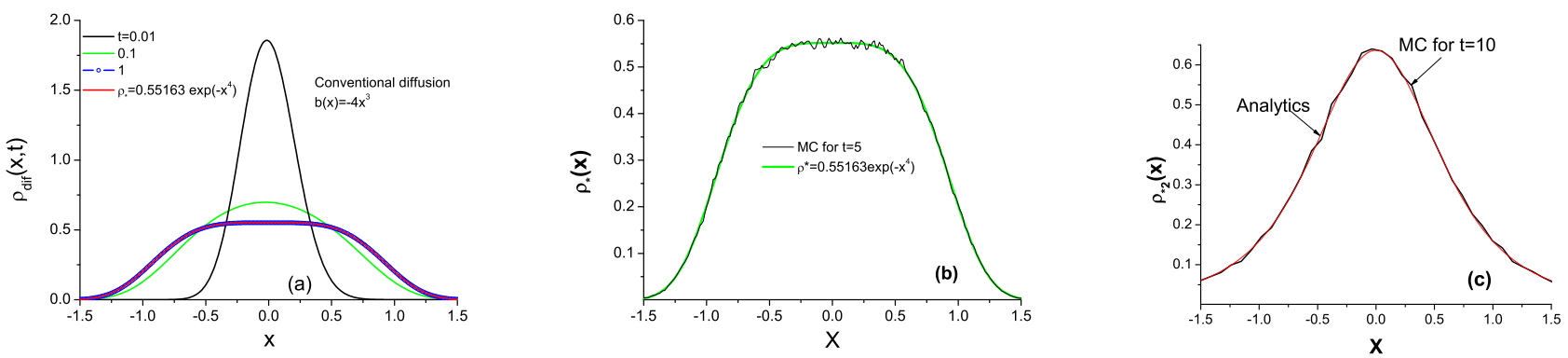

FIG. 6: Time evolution of a diffusion process with drift $b(x)=-4 x^{3}$. Panel (a) shows the pdf dynamics, obtained from FP equation. Panel (b) reports the comparison of path-wise MC simulation for $t=5$ with analytical result for asymptotic pdf. Simulated pdf has been intentionally left "noisy" (i.e. created with smaller number of sample trajectories) to reveal the coincidence with analytical curve. Panel (c) compares the MC result at time $t=10$ with the analytical result for $\rho_{* 2}(x)=$ $(2 / \pi) /\left(1+x^{2}\right)^{2}$. The simulated pdf has been intentionally left "noisy".

\section{Cubic drifts}

Let us consider a cubic function $b(x)=-4 x^{3}$ as an exemplary drift in the F-P equation. This function surely does not obey the growth condition 39, 40]: $|b(x)|+1 \leq$ $C(1+|x|)$. Standard arguments [4, 39] allow us to identify a stationary pdf associated with this drift function. It has an explicit Gibbs form $\rho_{*}=(1 / Z) \exp \left(-V_{*}\right)$, where $V_{*}=x^{4}$.

To get convinced that it is actually an asymptotic pdf of a well defined diffusion-type process, we address the time evolution of $\rho(x, t)$ that is started from "almost" Dirac delta pdf and propagated in accordance with the corresponding FPE. The resultant relaxation to the nonGaussian equilibrium pdf $\rho_{*}(x) \sim \exp \left(-x^{4}\right)$, is depicted in Fig. 6a.

This relaxation pattern has been directly path-wise confirmed by executing the MC simulation of c.a. $10^{5}$ Brownian paths, up to the time instant $t=50$. This allowed us to check the shape and stability of the corresponding pdfs. Fig. 6b reports a direct comparison between the simulated (up to time $t=5$ as for $t>5$ the corresponding pdfs are exactly the same) and analytic asymptotics $\rho_{*}(x)=0.55163 \exp \left(-x^{4}\right)$.

Our simulation shows, that a perfect agreement of two different generation methods for a given pdf extends to (arbitrarily) large times. Each of the transitional pdfs shown in Fig. [6a can be consistently reproduced trajectory-wise, i.e. by means the MC simulation whose record is stored and analyzed at each required time instant. In Fig. 6 b o we have intentionally allowed the simulated asymptotic pdf to be somewhat "noisy". That outcome is a consequence of not too large number of sample trajectories employed for the pdf approximation. Thus, on computer-assisted grounds, the time evolution of pdfs generated by FPE's with polynomial drifts, appears to be consistent.

\section{Cauchy family targeting}

In relation to the discussion of Section II, our simulations have shown that a proper choice of the drift function in the FPE, as $t \rightarrow \infty$ takes the initial $\delta$-function into any Lévy - stable distribution target, or the like (e.g. members of the Cauchy family). Accordingly, conventional diffusion-type processes may in principle give rise to "heavy-tailed" asymptotic pdfs. Thus, heavy tails are not an exclusive property of Lévy processes.

The only (and very substantial) difference between "normal" and fractional FPE asymptotic outcome is that in the fractional case Lévy flights may not relax to GibbsBoltzmann pdfs, [1]. In turn, diffusion modeling with a suitable external forcing admits a Gibbs form of asymptotic "heavy-tailed" pdfs.

Fig. 6r reports an analogous (F-P dynamics vs MC simulation) comparative procedure, in case when both Cauchy and Wiener drivers are responding to different external forces, while giving rise to a common asymptotic pdf. Cauchy dynamics does not produce the Gibbs pdf. However, the same pdf has the Gibbs form in the diffusive relaxation process.

We have directly checked that a target function belonging to Cauchy family (24), $\rho_{* 2}(x)$, is an asymptotic outcome of the diffusion process via path-wise simulation, with $b_{2, \text { diff }}(x)$ of Eq. (39). We have confirmed that the $\mathrm{MC}$ procedure (with check-out times $t=0,01, t=0,1$, $t=1$ and $t=8$ ) correctly reproduces the qualitative picture of the time evolution depicted in Fig. 4h. The ultimate outcome in the vicinity of the asymptotic pdf is reported in Fig. 6.6.

The very same reasoning may be adopted for exemplary jump-type processes, with Cauchy driver and polynomial drift functions. We note that relaxation properties and confinement of Lévy flights in various external potentials has received attention in the literature, see e.g. references in [1 $[3,7,9]$. For example, the CauchyLangevin dynamics has been analyzed for $\partial_{t} \rho=-|\nabla| \rho-$ 


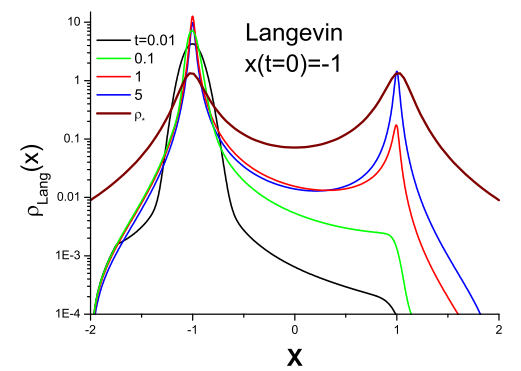

(a)

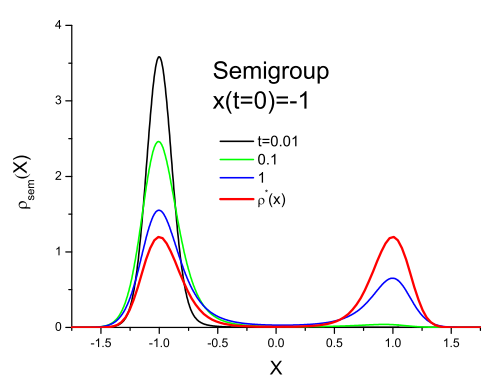

(d)

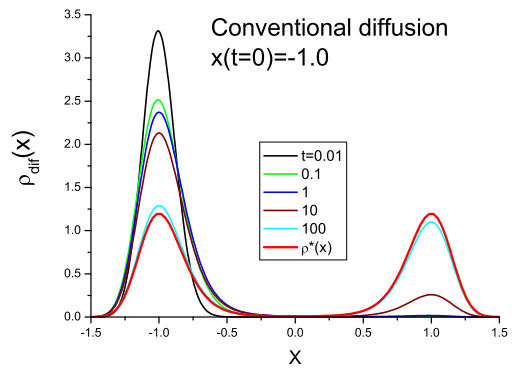

(g)

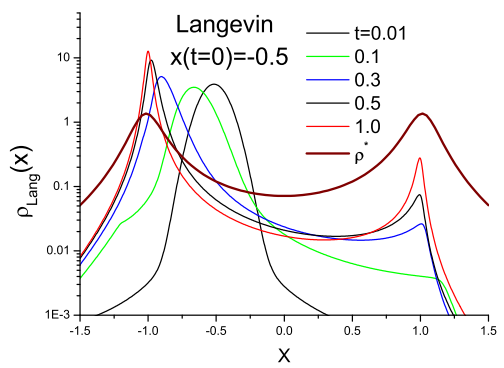

(b)

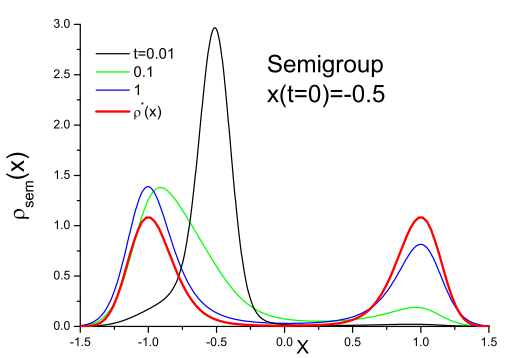

(e)

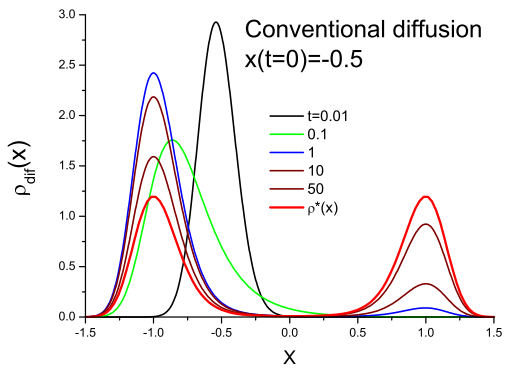

(h)

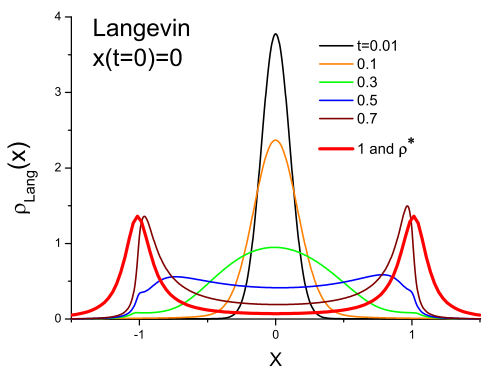

(c)

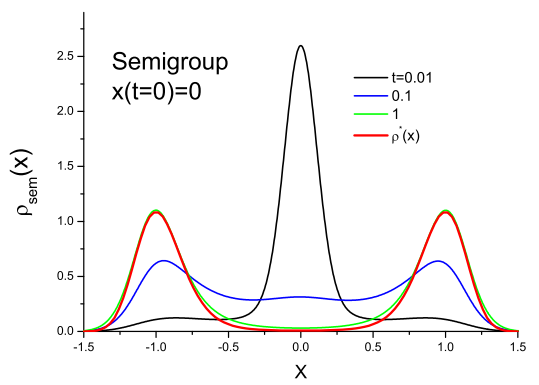

(f)

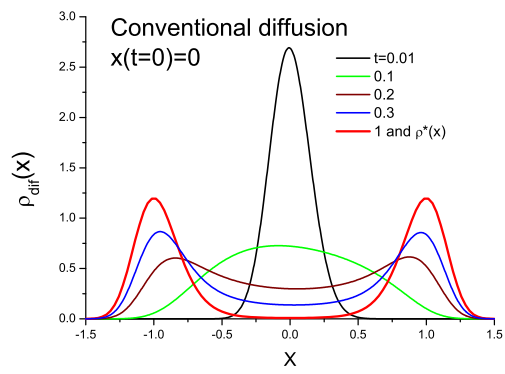

(i)

FIG. 7: The relaxation to the bimodal pdf (59) for three different type of processes: Langevin driven (panels (a) - (c)), semigroup driven (panels (d) - (f)) and Wiener driven (conventional FPE diffusion) (panels (g) - (i)). The initial Dirac delta-like pdf is located, respectively, at $x=-1$ (panels (a), (d), (g)), $x=-0,5$ (panels (b), (e), (h)) and $x=0$ (panels (c), (f), (i)). All three processes have different drift functions, corresponding to terminal pdf (59) as indicated in the text. On panels (a) and (b), the $\log$ scale is utilized for better visualization of time evolution of initial unimodal pdf to the terminal bimodal one.
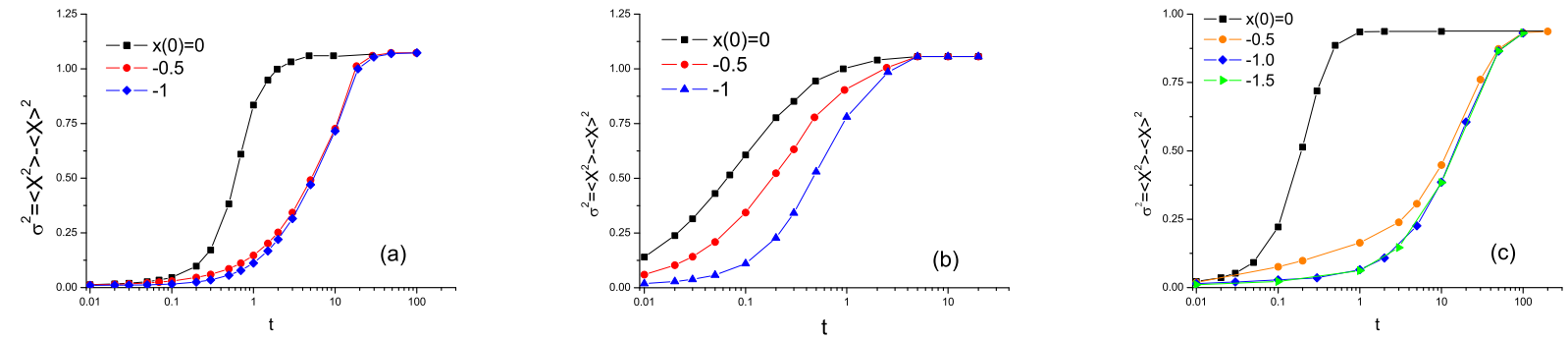

FIG. 8: The time evolution of variances for pdfs in Fig. 7 Panels (a), (b) and (c) correspond to Langevin driven, semigroup driven and conventional diffusion (Wiener driven) process respectively 
$\nabla(b \rho)$, with $b_{\text {jump }}(x)=-x^{3}$. The corresponding nonGibbsian invariant pdf reads $\rho_{*}(x)=1 / \pi\left(x^{4}-x^{2}+1\right)$, [7, 8].

At this point we can again invoke $\rho_{* 2}(x)$, this time regarded as an asymptotic target of the Cauchy-Langevin jump-type process with the drift function $b_{2, j u m p}(x)=$ $-x\left(x^{2}-3\right) / 8$ of section II.B. Both the resultant pdf evolution and the corresponding Monte Carlo simulation, in terms of jump sample paths, give consistent outcomes.

Concerning mathematical subtleties of the Cauchy semigroup dynamics, their well-definiteness comes from the properties of semigroup potentials. This issue has received due attention in Ref. [12], see also [8, 9].

\section{B. Relaxation time rates}

In recent years there has been continually growing interest in various theoretical random walk models, clearly motivated by the inefficiency of the standard Gaussian modeling paradigm. That refers to various sub-fields of physics, extending to chemistry, biology, biophysics and financial mathematics.

The classical concept of Brownian motion pervades the whole theory of stochastic processes. The pdf of a homogeneous Brownian motion solves a Fokker-Planck (actually, heat) equation and remains sweeping unless confined in a finite region or subject to external forces. Typically the time-evolving pdf, that is initially concentrated at (or about) a point, with the flow of time takes the Gaussian form, whose width grows in time as $t^{1 / 2}$. This diffusion processes is called the normal diffusion.

In a broad field of anomalous diffusions (and more general non-Gaussian processes), whose microscopic modeling may involve both Markovian and non-Markovian random dynamics, another property has been discovered: $<X^{2}(t)>\sim t^{\gamma}$, with $0<\gamma<2$. This dynamics is sweeping as well, hence precluding the existence of any well defined relaxation pattern (except for a possible relaxation to a uniform distribution, if the motion is spatially confined).

For a special class of non-Gaussian jump-type processes, first and second moments of the pdf may not exist at all. That happens in case Lévy - stable distributions. Due to long tails of the pdf, in the least their second (and all higher) moments are nonexistent. The existence of the first moment is granted only for a suitable subclass.

In the presence of external potentials the confinement (taming) of Lévy flights may occur, and quite in affinity with standard diffusion processes in external force fields, asymptotic invariant pdfs may be approached. The speed (time rate) of the corresponding relaxation processes is worth addressing and set against that arising in the sweeping motion.

Somewhat intriguing point in the anomalous transport is whether, often used terms like "subdiffusion" or "superdiffusion" may at all have meaning in connection with Lévy flights, under confining (i.e. relaxation) regimes.
Clearly, there always appears a transitional period during which the diffusion, jump-type or semigroup-driven process may be analyzed in terms of a time dependent variance (unless non-existent). This property is regarded as generic in relaxing to equilibrium diffusion processes.

Our purpose is to check whether one may expect any regularity in the time evolution of variances for diffusiontype and jump-type (Langevin and semigroup-driven) processes. That could possibly give useful hints towards their hierarchical classification with respect to relaxation time rates. May we talk about "slow", "fast" or "superfast" processes at all ?

Such hypothesis might look plausible on the basis of our previous analysis of relaxation scenarios whose asymptotic targets were pdfs from the Cauchy family, Eq. (11). However, the situation is not that simple and appears to be incongruent with naive expectations. This issue we shall discuss below.

\section{Relaxation to the Cauchy pdf $\rho_{* 1}$}

Our first observation pertains to the OrnsteinUhlenbeck-Cauchy process (OUC, Langevin-driven) and its semigroup-driven relative with a common target pdf $\rho_{* 1},[10$. It is seen form Fig. 4, that both the transitional behavior and the time needed to reach (almost, within the figure resolution limits) the asymptotic pdf, indicate that the semigroup dynamics is running somewhat slower as compared to Cauchy-Langevin-driven dynamics. Indeed, the transitional period of motion admits a direct analysis of the half-widths (second moments of $\rho_{* 1}$ are non existent, see above) time rate in terms of respective exponents of $t^{\gamma}$. Namely, for the proper OUC process, the transitional exponent equals $\gamma=0.58$, while in the semigroup-driven case, we have $\gamma=0.45$.

Both processes are of the jump-type and have no second moments. We note that the Cauchy-Langevin driving sets the $\rho(x, t)$ in the vicinity of the invariant one after time $t=8$, while the semigroup-driving needs $t=15$ to the same end. The diffusion scenario, according to Fig. 4r sets at $\rho_{* 1}$ after time $t=5$.

\section{Relaxation to the bimodal pdf}

Now we discuss various aspects of relaxation time rates for diffusion-type, Cauchy-Langevin driven and Cauchy semigroup-driven processes. All of them are started from "almost" Dirac delta pdfs (albeit localized about three different initial points, and all terminated at an asymptotic bimodal pdf, [10]

$$
\rho_{*}(x)=\frac{2 a\left(a^{2}+b^{2}\right)}{\pi} \frac{1}{\left(a^{2}+b^{2}\right)^{2}+2\left(a^{2}-b^{2}\right) x^{2}+x^{4}}
$$

Here, parameters $a$ and $b$ are, respectively, real and imaginary parts of complex roots of the cubic equa- 
tion $z^{3}+z-1 / 4=0$. Their approximate values are $a \simeq 0.118366$ and $b \simeq 1.0208$.

This exemplary bimodal pdf is an asymptotic distribution of the Cauchy-Langevin driven dynamics of $\rho(x, t)$ with the drift $b_{\text {jump }}=-\nabla V_{*}(x)$, where $V_{*}(x)=\left(x^{2}-1\right)^{2}$. The Cauchy-Langevin evolution of $\rho(x, t)$, that is started from three different locations $x=0, x=-0,5$ and $x=-1$ in the double well potential $V_{*}$, is depicted in Fig. 7, panels (a)-(c).

The data collected for Fig:7(a)-(c) allow to deduce the time-dependence of variances, in the transitional regime. They display $\sim t^{\gamma}$ behavior which depends on the initial pdf location data. Namely, for $x=-1$ and $x=-0,5$ we have respective exponents $\gamma=0.53$, while $x=0$ yields $\gamma=1$.

The Cauchy semigroup-induced evolution of $\rho(x, t)$, has been simulated under the very same (as in the Cauchy-Langevin case, Fig[7 (a)-(c)) initial data. The corresponding patterns of behavior are depicted in Fig 7. panels (d)-(f). In Fig. 8 we report the time evolution of respective variances. The $\sim t^{\gamma}$ behavior in the transitional regime is characterized by exponents: $\gamma=1$ for $x=-1, \gamma=0.53$ for $x=-0.5$ and $\gamma=0.2$ for $x=0$.

For completeness we have performed analogous simulations with the Wiener driver in action, i.e. for the conventional diffusion-type process that interpolates between the common (for all three types of processes) initial data and the terminal bimodal pdf, see e.g. also Ref. [43]. The results are shown on panels (g) - (i) of Fig[7. In Fig. 8 we also report the temporal behavior of related variances. The $\gamma$ exponents in the transitional regime read: for $x=-1.5$ and $x=-1$ we get $\gamma=0.75$, $x=-0.5$ corresponds to $\gamma=0.5$, while $x=0$ to $\gamma=1.2$.

The preceding analysis shows that standard classification of anomalous diffusion processes as "subdiffusions" or "superdiffusions" on the basis of the exponent $\gamma$, is invalid in the presence of external forces. Moreover, this exponent strongly depends on both the particular localization of the initial pdf (effectively, an initial position of a fictitious particle), with respect to the corresponding potential profile, and on the potential curvature.

\section{CONCLUSIONS}

The main message of the present paper is that, under suitable confinement conditions, the ordinary FokkerPlanck equation may generate a non-Gaussian heavytailed pdf (like e.g. Cauchy or more general Lévy stable distribution) as its long time asymptotics. That implies a continuous interpolation between an initial highly localized pdf (like Dirac $\delta$ - function) having all moments and the terminal heavy - tailed pdf, with only few (or none at all) moments in existence.

Since it is the fractional generalization of FPE which is customarily invoked to generate the same heavy-tailed pdfs, albeit with a very different choice of drift functions, we have uncovered an unexpected link between non-
Gaussian jump-type processes (inherent in the fractional FPE derivation) and ordinary diffusion processes that are based on the Gaussian (Wiener driver) paradigm.

In the present paper we have paid attention not only to random processes that have found their place in the literature, all stemming from different forms of Langevin modeling (here, only additive noise has been considered), but also to alternative jump - type scenarios based on the concept of the semigroup-driven dynamics, [8, 12]. This pattern of dynamical behavior has not been satisfactorily explored within the area of anomalous random transport.

To make our findings transparent, we have undertaken a direct numerical verification of them, by invoking two kinds of modeling. The first one amounts to solving numerically both ordinary (with the Laplace operator) and fractional FPE's with the drift functions selected to generate, the same for both, non-Gaussian (specifically Cauchy family (24)) pdfs. The second one presumes that one has the above drift functions at disposal. Then, we undertake a direct numerical modeling of random trajectories (sample paths) of the underlying stochastic process, both Brownian and of the jump-type. These trajectories have next been used to generate the associated pdfs which turn out to coincide with those obtained from numerical solution of both ordinary and fractional FPE's.

We note that a traditional interpretation of random data relies on the existence of at least two lower moments of involved probability distributions. Therefore, the case of Lévy flights could have been placed within this interpretational paradigm only under confining conditions. In such case, in view of the existence of second moments, one may address their time-dependence in transitional regimes, when the process is yet far from its equilibrium pdf. The above numerical solutions of the FPE and of its fractional analog permit us to investigate the time dependence of different moments (see Fig. 8) of the corresponding pdfs.

At this point we have passed to an approximation of the variance $\sigma^{2}$ by the power law $\sigma^{2} \sim t^{\gamma}$, with the hope that its validity would shed some light on the problem of assigning terms like "subdiffusion" or "superdiffusion" to anomalous transport processes. Namely, the time evolution of variances is often considered as a signature of subdiffusive, normal or superdiffusive behavior, typically considered with no bounds on the duration time of the process (unless set be experiment). Taken literally, all such motions would belong to the sweeping category, where the variance grows indefinitely.

In this connection, our analysis shows that (i) near initial $t \rightarrow 0$ the exponent $\gamma$ depends strongly on time, (ii) deeply in the asymptotic $t \rightarrow \infty$ regime $\gamma$ can be equated to 0 , (iii) in a transitional regime (i.e. for pdfs lying somewhere between initial and terminal ones) the exponent $\gamma$ happens to take almost constant positive values (observe e.g. almost straight lines in time dependence of variances in the middle parts of the corresponding plots in Fig. 8).

We have focused on possible signatures of (ab)normal relaxation patterns, expecting that in a transitional 
regime we might possibly identify an undoubtful subdiffusive or superdiffusive dynamics. Our answer is negative. We have confirmed that widely used terms "sub" and/or "superdiffusion" are rather vague and become meaningless for confined stochastic processes, likewise in the presence of Lévy and Wiener drivers. The pertinent exponent value is not indicative for the stochastic process in question, to justify its naming (sub-, normal, super-), because it depends strongly on the location (position) of the initial pdf relative to the drift potential profile, and on its curvature.

It appears that the above "anomaly" in the description of random motions appears to have much deeper foundations. One can alternatively use incompatible microscopic mechanisms to model an engineered asymptotic approach to the same a priori prescribed non-Gaussian target pdf.

If those pdfs are an outcome of a statistical analysis of experimental data, clearly a distinction between diffusive and jumping patterns of dynamical behavior appears not to be that sharp as commonly expected. On the other hand, a possible discrimination tool, e.g. the singleparticle/molecule experiments in the nano- or mesoscopic domain should be set under scrutiny, since microscopic features of motion may not be verifiable at current levels of precision/resolution in collecting the data. The specific stochastic model that would seemingly fit to the data, might possibly be not more than a lucky guess, to be invalidated in a more thorough analysis.

Another message worth spelling is that apparently divorced dynamical mechanisms (Langevin-Cauchy, Cauchy semigroup and diffusion-type process) may share common asymptotic pdfs. Contrary to the wide-spread belief, heavy-tailed probability distributions, casually associated with jump-type processes, may as well have Gaussian (e.g. diffusive) origins. That is possible if drift functions refer to properly functioning potentials, which we attribute to a cumulative effect of inhomogeneities of the environment). Their role would be to attenuate the casually considered counterbalancing of the Wiener driver, "normally" necessary to eliminate heavy tails of the resultant pdf.
[1] I. Eliazar and J. Klafter, J. Stat. Phys. 111, 739, (2003).

[2] S. Jespersen, R. Metzler and H. C. Fogedby, Phys. Rev. E 59, 2736, (1999)

[3] V. V. Janovsky et al., Physica A 282, 13, (2000)

[4] H. Risken, The Fokker-Planck equation, Springer-Verlag, Berlin, 1989

[5] A. A. Dubkov, B. Spagnolo and V. V. Uchaikin, Int. J. Bifurcations and Chaos, 18, 2549, (2008)

[6] A. Dubkov and B. Spagnolo, Fluct. Noise Lett. 5, L267, (2005)

[7] A. Dubkov, A. La Cognata and B. Spagnolo, J. Stat. Mech. P01002, (2009)

[8] P. Garbaczewski and V. A. Stephanovich, Phys. Rev. E 80, 031113, (2009).

[9] P. Garbaczewski, Physica A 389, 936, (2010).

[10] P. Garbaczewski and V. A. Stephanovich, Physica f A 389, 4419, (2010)

[11] J. C. Zambrini, Phys. Rev. A 35, 3631, (1987)

[12] P. Garbaczewski and R. Olkiewicz, J. Math. Phys. 41, $6843,(2000)$.

[13] E. W. Montroll and M. F. Shlesinger, J. Stat. Phys. 32, 209, (1983)

[14] J. N. Kapur and H. K. Kesavan, Entropy Optimization Principles with Applications, Academic Press, Boston, 1992

[15] J. Naudts, private communication

[16] P. Garbaczewski and V. A. Stephanovich, Open Syst. Inf. Dynamics, 17, No. 3, (2010)

[17] I. Bronstein et al., Phys. Rev. Lett. 103, 018912, (2009)

[18] C. Salomon et al., Europhys. Lett. bf 12, 683, (1990)

[19] S. Marksteiner, K. Ellinger and P, Zoller, Phys. Rev. A 53, 3409, (1996)

[20] H. Katori, S. Schlipf and H. Walther, Phys. Rev. Lett. 79, 2221, (1997)

[21] E. Lutz, Phys. Rev. A 67, 051402, (2003)

[22] E. Lutz, Physical Review Letters, 93, 190602, (2004)
[23] J. Jersblad at al., Phys. Rev. A 69, 013410, (2004)

[24] P. Douglas, S. Bergamini and F. Renzoni, Phys. Rev. Lett. 96, 110601, (2006)

[25] D. A. Stariolo, Phys. Lett. A 185, 262, (1994)

[26] C. Tsallis et al., Phys. Rev. Lett. 75, 3589, (1995)

[27] M. O. Caceres, Physica A 218, 471, (1995)

[28] P. A. Alemany and D. H. Zanette, Phys. Rev. E 49, R956, (1994)

[29] D. H. Zanette and P. A. Alemany, Phys. Rev. Lett. 75, 366, (1995)

[30] A. R. Plastino and A. Plastino, Physica A 222, 347, (1995)

[31] C. Tsallis and D. J. Bukman, Phys. Rev. E 54, R2197, (1996)

[32] L. Borland, Phys. Lett; A 245, 67, (1998)

[33] G. Kaniadakis and G. Lapenta, Phys. Rev. E 62, 3246, (2000)

[34] J. Naudts, Physica A 340, 32, (2004)

[35] M. Akimoto and A. Suzuki, J. Korean Phys. Soc. 40, 974, (2002)

[36] D. A. Kessler and E. Barkai, arXiv:1005.4737, (2010)

[37] A. Manor and N. M. Shnerb, Phys. Rev. Lett. 103, 030601, (2009)

[38] P. T. Landsberg (ed.), Problems in thermodynamics and statistical physics, Academic Press, Boston, 1971

[39] M. C. Mackey, Time's arrow: The origins of thermodynamic behavior, Springer-Verlag, Berlin, 1992

[40] K. Sobczyk, Stochastic differential equations, Kluwer, Dordrecht, 1991

[41] W. Horsthemke and R. Lefever, Noise-induced transitions, Springer-Verlag, Berlin, 1984

[42] N. G. van Kampen, Stochastic processes in physics and chemistry, Nort-Holland, Amsterdam, 1987

[43] D. Brockmann and I. Sokolov, Chem. Phys. 284, 409, (2002) 